\title{
On Dynamical Systems and the Minimal Surface Equation
}

\author{
by \\ Per Tomter \\ University of Oslo
}

\section{Introduction}

The relationship between the theory of dynamical systems and differential geometry is a long standing and profound one. It has largely been focused around the geodesic flow on the unit tangent bundle of a Riemannian manifold. One may recall early work by Poincaré and Birkhoff for the case of convex surfaces, Morse-theory, contributions by Hadamard, and, more recently, by Anosov, in the case of negatively curved manifolds - to register the decisive influence that has been asserted by this particular example in the development of the general theory of dynamical systems. Conversely, analysis of the geodesic flow has been useful to study problems in Riemannian geometry, one may point to the recent successful structure theory for Hadamard manifolds by Ballmann, Eberlein, Gromov, Schroeder and others. Here we will be concerned with a different, more unexpected role that dynamical systems have recently played in the very active field of partial differential equations that arise in Riemannian geometry, notably the minimal surface equation. Briefly, in the presence of a suitably chosen Lie group $G$ of isometries, one studies the $G$-invariant solutions. A simple case of this idea goes back to Delaunay's classification of rotationally invariant constant mean curvature surfaces ([D]); more recently, the celebrated work of Bombieri-de Giorgi-Giusti on the Bernstein problem may also be viewed in this context ([BGG]). The ideas were developed in a more systematic way as a general program of "equivariant geometry" in a seminal paper by W.Y. Hsiang and B. Lawson ([HL]), and has since been developed, espe- 
cially by Hsiang, to solve some long standing open problems in Riemannian geometry ([H1], [H2], [H3]).

In the case when the principal orbits of $G$ have codimension two in the manifold $M$, the reduced minimal equation in the orbit space $M / G$ becomes a dynamical system, in fact, for a suitably modified metric on $M / G$ we are again faced with the geodesic flow. Since $M / G$ has singular strata, where the curvature blows up, there are new features here, and some hard analysis is usually required to find closed solutions in each particular case. Most interesting solutions which have been found (minimal hyperspheres in various symmetric spaces, including counterexamples to the "spherical. Bernstein problem" posed by S.S. Chern , [H1], [H2], [T1]), have been obtained from geodesics in $M / G$ which are "closed" only in the more general sense that their end points are on singular strata.

After giving a brief survey of generalities we outline, in section 1 , the computation of the relevant orbital invariants for actions of suitable isometry groups on the projective planes over the complex numbers $\mathbf{C}$, the quaternions $\mathrm{H}$, and the Cayley numbers $C a$, respectively. In section 2 we investigate the resulting dynamical system, and in section 3 we prove the following:

Theorem: Let $\mathbf{C P}(2), \mathbf{H} P(2), C a(2)$ be the projective planes over $\mathbf{C}, \mathbf{H}$, and $C a$, respectively. In each case there exists an embedded closed minimal hypersurface of generalized torus type, namely:

(i) for $\mathrm{C} P(2): S^{1} \times S^{1} \times S^{1}$-type.

(ii) for $\mathrm{HP}(2): S^{1} \times S^{3} \times S^{3}$-type.

(iii) for $C a(2): S^{1} \times S^{7} \times S^{7}$-type.

This is an example of the type of question that has come up in Hsiang's program, and in this case the solutions are obtained by finding an honestly 
closed geodesic in the regular part of the orbit space. For dimension two (i.e. projective planes) the analysis is considerably simplified by the existence of a finite reflection group of symmetries in orbit space; in the case of the Cayley projective planes these arise from triality.

In the last section 4 we illustrate this by showing some solution curves obtained by numerical computations. These pictures suggest the existence of many further closed solutions; moreover, they appear compatible with orbital stability. The curvature of $M / G$ is positive, so, some closed geodesics may be expected to be elliptic. This raises the question of developing the theory of geodesic flow for this type of stratified manifolds (in particular, to which extent the Kolmogorof-Arnold-Moser theory is applicable to prove the existence of many closed solutions near a closed solution, possibly of singular type, as described above). In some cases many "closed" solutions of singular type have already been found by more ad hoc methods ([HT], $[\mathrm{HHT}]$ ), but the natural way in which these types of dynamical systems with singularities make their appearance, as well as the far-reaching results already obtained for specific cases, suggests further study of the general properties of such systems.

The computer computations were done in collaboration with Gil Bor at the University of California, Berkeley.

\section{1}

Let $G$ be a compact Lie group acting isometrically on a closed Riemannian manifold $M$. The orbit projection $\pi: M \rightarrow M / G$ is then a Riemannian submersion when restricted to the open, dense set $M^{0}$ of points on principal orbits; here we use the orbital distance metric $d s^{2}$ on $M^{0} / G$. To reduce the study of the minimal equation in $M$ to a problem in $M^{0} / G$, the only invariant needed from the geometry of the fibres of $\pi$ is the function $W$ on $M^{0} / G$ which records the volume of the orbits. For a curve $\gamma$ in $M^{0} / G$ the 
volume of $\pi^{-1}(\gamma)$ in $M$ is then the integral of $W$ along $\gamma$, or, equivalently, the arc length of $\gamma$ with respect to the modified metric $d s^{-2}=W^{2} d s^{2}$ on $M^{0} / G$. It follows that $\pi^{-1}(\gamma)$ is minimal in $M$ iff $\gamma$ is a geodesic in $\left(M^{0} / G, d s^{-2}\right)$; and the geodesic equation is as follows ([HL]):

$$
k(s)-\frac{d}{d \bar{n}} \ln W(\gamma(s))=0
$$

where $\gamma(s)$ is the curve parameterized by arc lenght relative to $d s^{2}, k$ is the curvature of $\gamma$, and $d / d \bar{n}$ is the directional derivative of $W$ in the normal direction of $\gamma$ compatible with a chosen orientation. If we now assume that $G$ has cohomogeneity two, i.e. $\operatorname{dim} M^{0} / G=2$, then $\pi^{-1}(\gamma)$ is a minimal hypersurface in $M$ iff $\gamma$ is a solution of (1.1). Hence, the invariants needed to perform the reduction in a concrete case is the orbit space with its orbital distance metric and the volume function $W$.

It is, of course, only feasible to compute these invariants in simple cases. It is straightforward for cohomogeneity two orthogonal actions on Euclidean spaces and spheres, and easy for complex and quaternionic projective spaces by lifting in the Hopf fibration (see below). For the isotropy action on compact rank 2 symmetric spaces the Cartan-Weyl theory will give these invariants: the orbit space is a flat Cartan polyhedron and the volume function $W$ is given in terms of the restricted roots by Weyl's volume formula for the relative case ([HHT]).

1. Complex projective space: $\mathbf{C P}(n)=U(n+1) / U(1) \times U(n)$. (See [HHT]).

Let $G=U(1) \times U(1) \times U(n-1)$ act on $S^{2 n+1} \subseteq \mathrm{C}^{n+1}$ in the standard way, the action of $U(1)$ sitting diagonally in $G$ defines the Hopf fibration $p: S^{2 n+1} \rightarrow \mathrm{CP}(n)$. Since the fibres of $p$ are totally geodesic, $p$ defines a one-to-one correspondence between $U(1)$-invariant minimal hypersurfaces of $S^{2 n+1}$ and minimal hypersurfaces of $\operatorname{CP}(n)$. Hence it is sufficient to study the orbit data for $S^{2 n+1}$. The $G$-orbits of $\mathrm{C}^{n+1}=\mathrm{C}+\mathrm{C}+\mathrm{C}^{n-1}$ 
are of the type $S^{1}(x) \times S^{1}(y) \times S^{n-3}(z)$, and are characterized by the three non-negative radii $x, y, z$. Hence the orbit space for the restricted action on $S^{2 n+1}$ is the spherical triangle $\left\{(x, y, z) \mid x^{2}+y^{2}+z^{2}=1, x, y, z \geq 0\right\}$. Introducing spherical polar coordinates: $x=\sin r \cos \theta, y=\sin r \sin \theta$, $z=\cos r$, we have:

(i) the orbit space is parameterized by $(r, \theta) \in\left[0, \frac{\pi}{2}\right] \times\left[0, \frac{\pi}{2}\right]$

(ii) the orbital distance metric is given by $d s^{2}=d r^{2}+\sin ^{2} r d \theta^{2}$.

(iii) $W(r, \theta)=x y z^{2 n-3}=\frac{1}{2} \sin ^{2} r \cos ^{2 n-3} r \sin 2 \theta$.

(Notice that we may normalize the volume function by any chosen constant factor.)

2. Quaternionic projective space: $\mathrm{HP}(n)=S p(n+1) / S p(1) \times S p(n)$.

By a similar argument with $G=S p(1) \times S p(1) \times S p(n-1)$ and lifing the $G$-action in the Hopf fibration $S^{3} \rightarrow S^{4 n+3} \rightarrow H P(n)$, one obtains:

the orbit space is given as in (i), (ii) above;

the volume function $W(r, \theta)=\frac{1}{8} \sin ^{6} r \cos ^{4 n-5} r \sin ^{3} 2 \theta$.

3. The Cayley projective plane: $C a(2)=F_{4} / \operatorname{Spin} 9$.

In this case there does not exist any "Hopf-fibration" $S^{7} \rightarrow S^{23} \rightarrow \mathrm{Ca}(2)$, and it is a more challenging task to comute the orbitral invariants. We refer to a forthcoming paper ([T3]) for the details of this; the methods developed there may also be useful in more general symmetric spaces. All three cases are treated uniformly in [T3] as follows: $M$ is a two-point homogeneous space, hence the orbit space under the isotropy action may be represented by a geodesic segment from a point $P$ to the cut locus, represented by $A$; we may parameterize this segment by $r \in\left[0, \frac{\pi}{2}\right] . G$ is the subgroup of the isotropy group $G_{P}$ that fixes a point on the cut locus; then $G$ acts on $M$ 
with cohomogeneity two. The $G_{P}$-orbits corresponding to interior points on $P A$ are Berger-type spheres. Their isotropy representations have two irreducible components; hence their homogeneous metrics are determined by two scaling factors along $P A$, which are well-known to be $\frac{1}{2} \sin 2 r$ and $\sin r$. They are obtained by studying the lengths of suitable Killing-Jacobi fields along the geodesic. In case 3 we have $G_{P}=\operatorname{Spin} 9, G=\operatorname{Spin} 8$, the principal $G_{P}$-orbits are of type $\operatorname{Spin} 9 / \operatorname{Spin} 7=S^{15}$; the volumes of these geodesic spheres varies with $r$ as $2^{-7} \sin ^{7} 2 r \sin ^{8} r$. Under the $G$ action each $G_{P}$-orbit has one-dimensional orbit space, which may again be taken as a geodesic segment parameterized by $\theta \in\left[0, \frac{\pi}{2}\right]$. The fact that the $G_{P}$-orbits are not canonically reductive as homogeneous spaces makes computations rather complicated. In case 3 the orbits are of type $S^{7} \times S^{7}$, with a complicated metric (spheres not orthogonal). However, since $G$ still defines an ample space of Killing fields, it turns out to be possible to compute the scaling of the volume of $G$-orbits along the geodesic segment $\theta \in\left[0, \frac{\pi}{2}\right]$, we refer to [T3] for the detailswof this. The orbit space is given by the same spherical triangle as in (i), (ii) above, the volume function is given by $W(r, \theta)=2^{-7} \sin ^{14} r \cos ^{7} r \sin ^{7} 2 \theta$.

Remark. This is the same result as one would obtain formally by assuming the existence of a "Hopf-fibration" $S^{7} \rightarrow S^{23} \rightarrow \mathrm{Ca}(2)$ where the $G$-action could be lifted.

\section{2}

Let $P(a+1)$ be the projective plane over $\mathrm{C}$ for $a=1$, over $\mathrm{H}$ for $a=3$, and over $C a$ for $a=7$. Let $\Delta$ be the intersection of the unit sphere with the first octant in $\mathbf{R}^{3}$, parameterized by spherical polar coordinates $(r, \theta)$. We summerize some results from the last section. 
Proposition 1. Let $M=P(a+1)$, let $P \in M$, and let $A$ be the first point on the cut locus along a geodesic from $P$. Let $G_{P}$ be the isotropy group of $P$, and let $G$ be the stabilizer subgroup of $G_{P}$ at $A$. Then the orbit space $M / G$, considered in the orbital distance metric, is isometric to $\Delta$. The volume function is given by $W(r, \theta)=\sin ^{2 a} r \cos ^{a} r \sin ^{a} 2 \theta$, when suitably normalized).

We will now use the orbital distance metric $d s^{2}=d r^{2}+\sin ^{2} r d \theta^{2}$ unless specified otherwise. Let $\gamma(s)$ be a curve in int $\Delta$ parameterized by arc length, and let $\alpha$ be the angle between the tangent vector $\dot{\gamma}=\dot{r} \frac{\partial}{\partial r}+\dot{\theta} \frac{\partial}{\partial \theta}$ and $\frac{\partial}{\partial r}$ i.e. $\dot{r}=\cos \alpha, \dot{\theta}=\sin \alpha \sin ^{-1} r$.

Proposition 2. Let $\gamma(s)$ be a curve in int $\Delta$ as above. Then $\pi^{-1}(\gamma)$ is a minimal hypersurface in $M$ iff $\gamma$ satisfies the following:

$$
\text { (*): } \quad \begin{aligned}
& \dot{r}=\cos \alpha, \quad \dot{\theta}=\sin \alpha \sin ^{-1} r, \\
& \dot{\alpha}=K_{r} \sin \alpha+2 a \cos \alpha \sin ^{-1} r \cot 2 \theta,
\end{aligned}
$$

where $K_{r}=a \tan r-(2 a+1) \cot r$.

Proof: The normal of $\gamma$ is $\bar{n}=-\sin \alpha \frac{\partial}{\partial r}+\cos \alpha \sin ^{-1} r \frac{\partial}{\partial \theta}$, hence $\frac{d}{d \bar{n}} \ln W=$ $-2 a \sin \alpha \cot r+a \sin \alpha \tan r+2 a \cos \alpha \sin ^{-1} r \cot 2 \theta$. Furthermore, the curvature of $\gamma$ in $\Delta$ is easily computed by Liouville's formula to be $\dot{\alpha}+\cos r \dot{\theta}$, and $(*)$ follows by substitution into (1.1)

q.e.d.

Proposition 3. Let $S_{3}$ be the symmetric group on three letters, which acts on the orbit space by reflections across the bisectors of $\Delta$. Then $S_{3}$ is a symmetry group for the dynamical system (*).

Proof. These arise from the isometry groups on $M$, for example, in the case of $\mathrm{CP}(2)=U(3) / U(1) \times U(1) \times U(1)$ from the Weyl group permuting the three $U(1)$ 's. In case $M=C a(2)$ this comes from the triality principle: in this case the three edges of $\Delta$ are the fixed point sets of the three nonconjugate subgroups of Spin 7 type in Spin 8 , which are permuted by the 
triality outer automorphisms. These outer automorphisms of Spin 8 may in fact be represented as restrictions of conjugations by elements of $F_{4}$, and define isometries (see [T3]).

Corollary 1. Let $\gamma(s)$ be a solution curve for $(*)$ which intersects two bisectors of $\Delta$ orthogonally. Then $\gamma(s)$ may be extended to a periodic solution curve.

Proof. This follows from Proposition 3 by repeated reflectiosn across bisectors.

Proposition 4. The following curves are solutiosn for (*):

(a) $r=r_{0}=\operatorname{Arctan} \sqrt{\frac{2 a+1}{a}}, \alpha= \pm \frac{\pi}{2}$ (equator).

(b) $\theta=\theta_{0}=\frac{\pi}{4}, \alpha=0$ or $\alpha=\pi$ (meridian).

(c) $\sin r \sin \theta=\sqrt{\frac{a}{3 a+1}}$ or $\sin r \cos \theta=\sqrt{\frac{a}{3 a+1}}$.

(d) $\sin r \sin \theta=\cos r$ and $\sin r \cos \theta=\cos r$ (bisectors).

Proof. (a) and (b) are verified by substitution, (c) and (d) are images of (a) and (b) respectively by reflections from $S_{3}$.

Remark 1. The equator solution (a) is the geodesic sphere in $M$ around the point $r=0$ with maximal volume. Notice that $r=r_{0}$ is a "closed geodesic of singular type".

Remark 2. It follows that any other solution curve which goes from an interior point of one boundary arc to one on another boundary arc of $\Delta$ must also be the projection of a minimal hypersphere in $M$. Hence the existence of such solution curves is central to the uniqueness aspect of Bernstein-type problems, they have been investigated in great detail ([H1,3], [T1], [HT]). 


\section{3}

In this section we do the analytical work needed to prove the existence of the desired closed solution curves in int $\Delta$.

Proposition 5. Let $\gamma(s)=(r(s), \theta(s), \alpha(s))$ be a solution curve for (*). Then we have:

(a) a critical point for $\theta(s)$ is a local maximum (resp. local minimum) in the region $\theta>\frac{\pi}{4}$ (resp. $\left.\theta<\frac{\pi}{4}\right)$

(b) a critical point for $r(s)$ is a local maximum (resp. local minimum) in the region $r>r_{0}$ (resp. $r<r_{0}$ )

(c) a critical point for $\theta(s)$ is non-degenerate except for the meridian solution $\theta=\frac{\pi}{4}$, a critical point for $r$ is non-degenerate except for the equator solution $r=r_{0}$.

Proof: By computation we get: $\ddot{\theta}=2 a \sin ^{-2} r \cos ^{2} \alpha \cot 2 \theta$ when $\dot{\theta}=0$, and $\ddot{r}=-K_{r} \sin ^{2} \alpha$ when $\dot{r}=\cos \alpha=0$.

q.e.d.

Proposition 6. For each interior point $p$ of an edge of $\Delta$ there exists a unique solution of $(*)$ with initial point at $p$. The initial direction of this solution is orthogonal to the edge.

Proof: This can be seen by some local analysis of $(*)$ near the boundary and an argument using formal power series expansion, see [HH ], [T2].

Proposition 6. Let $O$ be the intersection point of the three bisectors of $\Delta$, then the coordinates of $O$ are $(r, \theta)=\left(\operatorname{Arctan} \sqrt{2}, \frac{\pi}{4}\right)$. Let $B$ be the corner of $\Delta$ given by $(r, \theta)=\left(\frac{\pi}{2}, \frac{\pi}{2}\right)$. Then the angle $\alpha$ of the bisector going into $B$ is in the interval $\left[\frac{\pi}{4}, \frac{\pi}{2}\right]$. 
Proof: Straightforward.

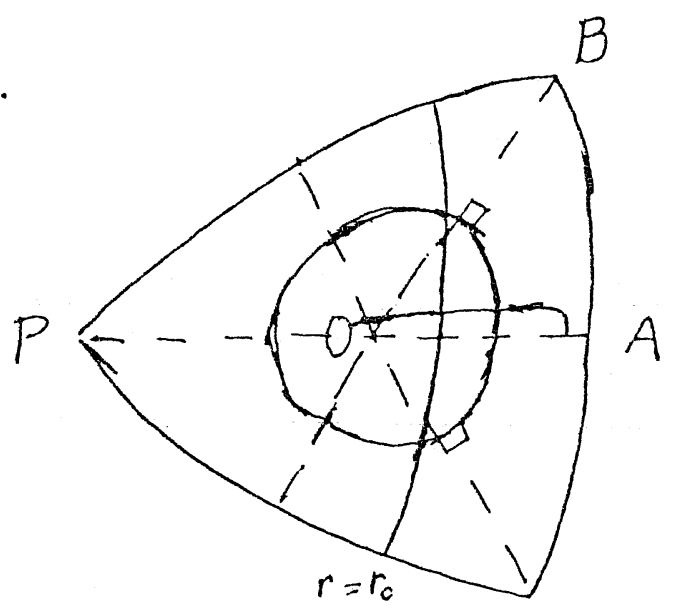

Definition: Let $\gamma_{b}(s)=\left(r_{b}(s), \theta_{b}(s), \alpha_{b}(s)\right)$ be the solution curve of $(*)$ with initial conditions $r_{b}(0)=b, \theta_{b}(0)=\frac{\pi}{4}, \alpha_{b}(0)=\frac{\pi}{2}$. Let $s_{b}$ be the smallest possible $s$ such that $\dot{\theta}\left(s_{b}\right)=0$, i.e. the first local maximum for $\theta_{b}(s)$.

Theorem. There exists a $b \in\left(r_{0}, \frac{\pi}{2}\right)$ such that $\gamma_{b}(s)$ defines a simple, closed solution curve of $(*)$ in int $\Delta$.

Proof: By Corollary 1 it is sufficient to show that there exists a $b \in\left(r_{0}, \frac{\pi}{2}\right)$ and a positive $d$ such that $\gamma_{b}(s), s \in[0, d]$ is a simple arc in the triangle $O A B$ (geodesic triangle in the $d s^{-2}$-metric), intersecting the bisector segment $O B$ orthogonally. The equator solution $\gamma_{\mathrm{ro}}$ intersects the bisector $O B$ at an angle in $\left(0, \frac{\pi}{2}\right)$, by continuous dependance on initial conditions this also holds for $\gamma_{b}, b \in\left(r_{0}, r_{0}+\varepsilon\right)$ when $\varepsilon$ is sufficiently small. It also follows that this intersection then occurs before $s=s_{b}$. Since the bisector and $\gamma_{b}$ are both solutions of (*), they never become tangent, and it follows that the first intersection point must persist as $b$ varies in $\left(r_{0}, \frac{\pi}{2}\right)$. (Notice that they cannot intersect at the boundary edge, by Proposition 6.) Furthermore, as long as the intersection occurs before $s=s(b)$, it must be on the segment $O B$. Assume that it should occur after $s=s\left(b_{1}\right)$ for some $b_{1}$, then, by continuity, it must occur exactly at $s=s\left(b_{2}\right)$ for some $b_{2} \in\left(r_{0}, b_{1}\right)$. But 
then, since $\alpha_{b_{2}}\left(s\left(b_{2}\right)\right)=\pi$ and by Proposition 6, the intersection angle between $\gamma_{b_{2}}$ and $O B$ is in $\left(\frac{\pi}{2}, \frac{3 \pi}{4}\right)$. From continuity it would then follow that $\gamma_{b}$ intersects $O B$ orthogonally for some $b \in\left(r_{0}, b_{2}\right)$, and we would be done.

Hence, for the remainder of the proof we may assume that $\gamma_{b}$ has its first intersection with $O B$ for some $s<s(b)$, whenever $b \in\left(r_{0}, \frac{\pi}{2}\right)$. We wish to consider $b$ close to $\frac{\pi}{2}$. Let $\bar{r}=-r$, from $(*)$ we get: $\frac{d \alpha}{d \bar{r}}=$ $K_{\bar{r}} \tan \alpha+2 a \sin ^{-1} r \cot 2 \theta$, hence $\frac{d \alpha}{d \bar{r}}>K_{\bar{r}} \tan \alpha$ in the region under consideration; furthermore $K_{\bar{r}} \tan \alpha>0.9 a \tan \bar{r} \tan \alpha$ for $r \in\left(r_{1}, \frac{\pi}{2}\right)$, with $r_{1}=\operatorname{Arctan} \sqrt{20+\frac{10}{a}}$. So in this region we now have $\frac{d \alpha}{d \bar{r}}>0.9 a \tan \bar{r} \tan \alpha$. The solution of the differential equation $\frac{d \alpha}{d \bar{r}}=0.9 a \tan \bar{r} \tan \alpha$ with the initial condition $\alpha(-b)=\frac{\pi}{2}$ is $\sin \alpha=\cos ^{0.9 a} b \cos ^{-0.9 a} \bar{r}$, comparing with this we may conclude that $\sin \alpha_{b}<\cos ^{0.9 a} b \cos ^{-0.9 a} \bar{r}_{b}$ as long as $r_{b}>r_{1}$. Hence, for a given positive $\varepsilon$, we get $\sin \alpha_{b}<\frac{e}{2}$ at $r=r_{1}$ by choosing $b$ sufficiently close to $\frac{\pi}{2}$. This shows that such $\gamma_{b}$ must turn sharply to $\alpha_{b} \in(\pi-\varepsilon, \pi)$ before $r=r_{1}$, and continues with $\alpha_{b} \in(\pi-\varepsilon, \pi)$ at least until $r=r_{0}$, this because by $(*) \dot{\alpha}_{b}$. is positive in this region, and by assumption $\alpha_{b}$ does not reach $\pi$ (corresponding to $s=s(b)$ ) before crossing $O B$, say at $s=6 s$. Furthermore, unless $\alpha_{b}$ reaches a local maximum before $s={ }_{b} s$, the same holds until that crossing, for $\varepsilon$ sufficiently small $\gamma_{b}$ then crosses $O B$ with angle in $\left(\frac{\pi}{2}, \frac{3 \pi}{4}\right)$, and we are done by the previous continuity argument. The final possibility that $\alpha_{b}$ reaches a local maximum before $s=b s$ would give $\dot{\alpha}_{b}=K_{r_{b}} \sin \alpha_{b}+2 a \cos \alpha_{b} \sin ^{-1} r_{b} \cot 2 \theta_{b}=0$, in this range this would imply that $\theta_{b}$ at such a point would approach zero as $\varepsilon \rightarrow 0$. In that case the initial conditions of $\gamma_{b}$ at $r_{b}=r_{0}$ approach those of the meridian solution $\theta=\frac{\pi}{4}, \alpha=\pi$ as $\varepsilon \rightarrow 0$, so continuous dependance on initial conditions would again imply that $\alpha_{b}$ stays close to $\pi$ until intersection with $O B$.

q.e.d. 
In this section we reproduce some solution curves $\gamma_{b}$ which were numerically obtained on a computer for the case of the Cayley projective plane, $a=7$. We remark that the pictures suggest the existence of many more nonsimple, closed solution curves. Moreover, small perturbations of the initial conditions of some of these appear to produce solution curves that project into a ring-shaped domain around the closed solution, which contains other closed solutions of higher period.

Recalling that this is the geodesic flow of $\left(\Delta, d s^{-2}\right)$, it is Hamiltonian, and the characteristic multipliers of a closed orbit are either $\lambda, \lambda^{-1}$, with real $\lambda,|\lambda|>1$ (hyperbolic case) or $e^{i \alpha}, e^{-i \alpha}$ with real $\alpha$ (elliptic case [AM]). The curvature of $\left(\Delta, d s^{-2}\right)$ is computed to be

$K=\sin ^{-(2 a+1)} r \cos ^{-a} r \sin ^{-a} 2 \theta\left(2 a \sin ^{-1} r \sin ^{-2} 2 \theta+(a+1) \sin r+a \tan r+\right.$ $\left.a \tan ^{2} r \sin r\right)>0$, hence elliptic periodic orbits are not unexpected. Analysis of the Poincare map is of interest since periodic points correspond to new closed minimal hypersurfaces in $M$. In the generic elliptic case the Moser twist stability theorem gives the existence of invariant tori containing periodic solutions of arbitrarily large period. The pictures would fit in with ring-shaped domains obtained as projections on the configuration space of such invariant tori, there has not been any study of this aspect so far. More interesting might be the case of singular closed orbits, (as the equator $\gamma_{r_{0}}$ ). Towards this, we only make the following observation:

Proposition 7. There is a well-defined Poincaré-map for a closed orbit $\gamma$ of singular type.

Proof: We consider $\gamma$ as defined globally by reversing direction from a boundary point; i.e. we oscillate back and forth along $\gamma$, with a discontinuity jump of $\pi$ for $\alpha$ at each boundary intersection. The only problem is to establish continuous dependance on initial conditions for solutions starting 
out near $\gamma$, also beyond $\gamma$ 's intersection with the singular boundary arc. This is non-trivial, although one can prove (with estimates similar to the proof of the theorem in section 3) that such a curve must follow $\gamma$ towards the boundary, turn sharply there, and start out again with initial data close to $\gamma$ on the way back. However, as we get closer to the boundary, there is no control over the Lipschitz constants of $(*)$. Nevertheless it is known that continuous dependance on initial conditions does persist past intersections with the boundary, for a proof, see [HT].

For example, in the case of the standard sphere, one could consider the possibility of using the Poincaré map of singular closed solutions to obtain nearby minimal closed hypersurfaces. If they correspond to singular closed solution curves, these would be hyperspheres. (In the case of the equator hypersphere, however, by an extrinsic rigidity theorem ([S]), it is known that there does not exist $C^{1}$-close minimal hyperspheres.) Although the particular question of existence of infinitely many minimal hyperspheres has already been treated successfully both for spheres ([HT]) and projective spaces ([T3]), it appears that the Hamiltonian viewpoint could be relevant for further study. This might especially apply to generic density properties of closed minimal hypersurfaces with respect to the cohomogeneity two metrics on $M$ respecting $G$. 

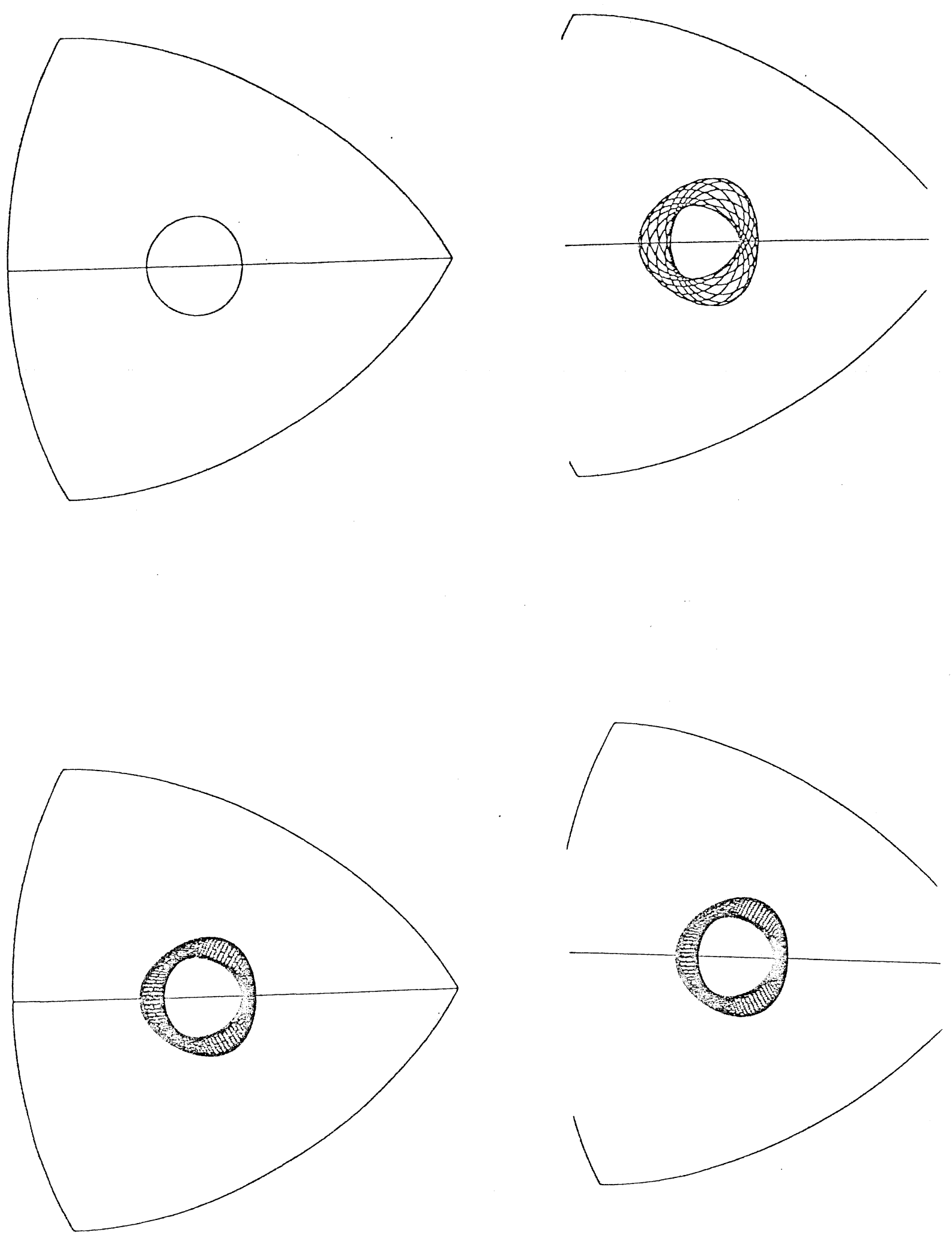


$$
8
$$




\section{References}

[AM] Abraham, R., and Marsden, J. E.: Foundations of Mechanics, 2. ed. The Benjamin/Cummings Publ. Co. (1978).

[BBG] Bombieri, E., de Giorgi, E., and Giusti, E.: Minimal cones and the Bernstein problem. Ivent. Math. 7 (1969), 243-268.

[D] Delaunay, C.: Sur la surface de révolution dont la courbure moyenne est constante, J. Math. Pures. Appl, Sér. 1.6., (1841), 309-320.

[H1] Hsiang, W. Y.: Minimal cones and the spherical Bernstein problem I. Ann. of Math. 118 (1983), 61-73.

[H2] Hsiang, W. Y.: Minimal cones and the spherical Bernstein problem II. Invent Math. 74 (1983), 351-369.

[H3] Hsiang, W. Y.: New examples of contant mean curvature immersions of $(2 k-1)$-spheres into Euclidean $2 k$-spaces. Ann. of Math. 117 (1983), $609-625$.

[HH] Hsiang, W. T., and Hsiang, W. Y.: On the existence of codimension one minimal spheres in compact symmetric spaces of rank 2, II. J. Diff. Geom., 17 (1982), 582-594.

[HHT] Hsiang, W. T., Hsiang, W. Y., and Tomter, P.: On the existence of minimal hypersphers in compact symmetric spaces. Ann. Scient. E.N.S., Vol. 21, (1988), 287-305.

[HL] Hsiang, W. Y., and Lawson, H. B.: Minimal submanifolds of low cohomogeneity. J. Diff. Geom., 5, (1970), 1-37.

[HT] Hsiang, W. Y., and Tomter, P.: On minimal immersions of $S^{n-1}$ into $S^{n}(1), n \geq 4$. Ann. Scient. E.N.S., Vol. 20 (1987), $201-214$. 
[S] Simons, J.: Minimal varieties in Riemannian manifolds. Ann. of Math. (2), 88 (1986), 62-105.

[T1] Tomter, P.: The spherical Bernstein problem in even dimensions and related problems. Acta Math. Vol. 158 (1987), 189-212.

[T2] Tomter, P.: Existence and uniqueness for a class of Cauchy problems with characteristic initial manifolds. J. Diff. Equations, 71 (1988), 19.

[T3] Tomter, P.: Minimal hyperspheres in two-point homogeneous spaces, to appear. 